
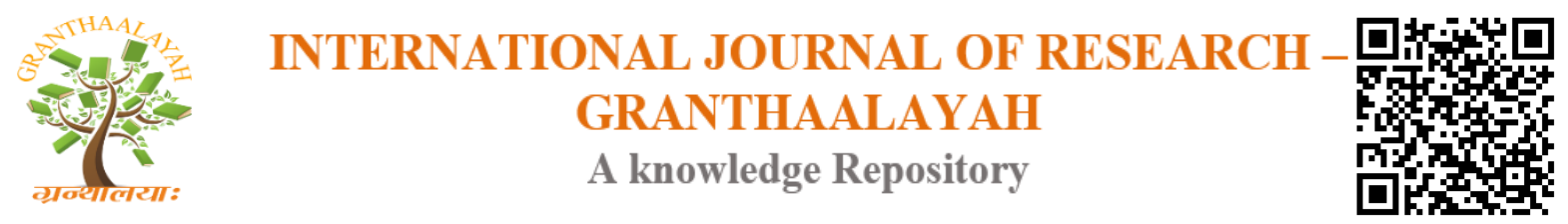

Social

\title{
DYNAMIQUE FAMILIALE EN MATIÈRE DE SANTÉ: ENTRE TRADITIONALISME ET MODERNISME CHEZ LES ABBEY, AU SUD DE LA CÔTE D'IVOIRE
}

\author{
Traore Kassoum ${ }^{1}$, Fofana Memon ${ }^{2}$ \\ ${ }^{1,2}$ Université Péléforo Gon Coulibaly de Korhogo (Côte d'Ivoire) UFR des sciences \\ sociales/Département de Sociologie
}

\begin{abstract}
Background: In Côte d'Ivoire, since the advent of the crisis of the HIV and AIDS epidemic, the structural adjustment policy of the 1980s and the end of the policy of total free healthcare for the majority of the population, the strategies within families for health emerged. In order to heal, the therapeutic routes within families are made by symbolic practices based on traditionalism, modernism and the juxtaposition of traditionalism and modernism.

Method: A qualitative study made it possible to collect data from semi-structured interviews with seven (07) families in which 22 key people were interviewed and ten (10) sellers of medicines (including 05 street drug sellers called "cheaper" and 05 traditional healers for medicinal plants). Also, a detailed documentary exploitation and a participant observation for a period of eight (8) months structured by an observation grid formalized in a notebook were conducted. These data were analyzed by taking into account endogenous and exogenous knowledge including the symbolic practices of structuring health within families and this community.

Result: The study shows that therapeutic dynamics within families are dominated by curative care practices with a juxtaposition of mobilizable care (modern and traditional medicine, selfmedication). This domination of the curative on the preventive is intended to anesthetize the health or therapeutic awareness of the patient and his family. In addition, the study found that the pharmaceutical basket is largely structured therapeutic products housed in the tradition model and therapeutic products under the "common sense" (street drug). The consequence of all these health practices within families is the observation of the death of the individual by an easily curable disease.

Conclusion: The present study was conducted in the Abbey community, an ethnic group in the southern region of Côte d'Ivoire, an area strongly marked by the colonization. The objective was to understand the social dynamics that structure the therapeutic practices and perceptions for the health care and preservation of the members of this community based on the health results obtained. In analysis, the remoteness of individuals from preventive therapeutic practices advocated by the health authorities is part of the actor strategy that Michel Crozier (1977) discusses, either to circumvent the financial expenses, or to give meaning to an event not judged too unfavorable for them or to reach a socio-cultural order not communicable.
\end{abstract}

\section{Résumé}


Contexte : En Côte d'Ivoire, depuis l'avènement de la crise de l'épidémie du VIH et du Sida, de la politique d'ajustement structurel des années 1980 et de l'arrêt de la politique de gratuité totale ${ }^{1}$ de soins de santé pour la majorité de la population, les stratégies à l'intérieur des familles en matière de santé ont émergées. Pour se soigner, les itinéraires thérapeutiques à l'intérieur des familles sont fabriqués par des pratiques symboliques fondées tantôt sur le traditionalisme, tantôt sur le modernisme, tantôt sur la juxtaposition du traditionalisme et du modernisme.

Méthode : Une étude qualitative a permis de collecter les données à partir des entretiens semistructurés auprès de de sept (07) familles au sein desquelles 22 personnes clés ont été interrogées et dix (10) vendeuses des médicaments (dont 05 vendeuses de médicaments de la rue dits « moins chers » et 05 tradipraticiens pour les plantes médicinales). Aussi, une exploitation minutieuse documentaire et une observation participante pour une période de huit(8) mois structurée par une grille d'observation formalisée dans un cahier ont été menées. Ces données ont été analysées en prenant en compte les connaissances endogènes et exogènes incluant les pratiques symboliques de structuration de la santé au sein des familles et de cette communauté.

Résultats: Il ressort de l'étude que la dynamique thérapeutique à l'intérieur des familles est dominée par les pratiques curatives de soins avec une juxtaposition des soins mobilisables (médecine moderne et traditionnelle, l'automédication). Cette domination du curatif sur le préventif est destinée à anesthésier la conscience sanitaire ou thérapeutique du malade et de sa famille. En outre, l'étude a révélé que le panier pharmaceutique est largement structuré de produits thérapeutiques logés dans le modèle de la tradition et des produits thérapeutiques relevant du "sens commun" (médicament de la rue). La conséquence qui découle de toutes ces pratiques de santé à l'intérieur des familles est l'observation du décès de l'individu par une maladie facilement curable. Conclusion: La présente étude a été réalisée dans la communauté des Abbey, un groupe ethnique dans la région sud de la Côte d'Ivoire, zone fortement marquée par la colonisation. L'objectif était d'appréhender la dynamique sociale qui structure les pratiques thérapeutiques et les perceptions pour les soins de santé et de préservation des membres de cette communauté à partir des résultats de santé obtenus. A l'analyse, l'éloignement des individus des pratiques thérapeutiques préventives prônées par les autorités sanitaires fait partie de stratégie d'acteur dont parle Michel CROZIER $^{2}$ (1977), soit pour contourner les dépenses financières, soit pour donner sens à un évènement jugé pas trop défavorable pour eux ou soit pour atteindre un ordre socio-culturel non communicable.

Mots clé: famille, santé, modernisme et traditionalisme, itinéraires thérapeutiques, médicaments de la rue

Keywords: Family; Health; Modernism and Traditionalism; Therapeutic Itineraries; Street Drugs.

Cite This Article: Traore Kassoum, and Fofana Memon. (2018). "DYNAMIQUE FAMILIALE EN MATIÈRE DE SANTÉ: ENTRE TRADITIONALISME ET MODERNISME CHEZ LES ABBEY, AU SUD DE LA CÔTE D'IVOIRE." International Journal of Research Granthaalayah, 6(4), 36-49. https://doi.org/10.29121/granthaalayah.v6.i4.2018.1474.

\footnotetext{
${ }^{1}$ Pour les soins du paludisme, la gratuité est pratiquée pour les femmes enceintes et les enfants de 0-5 ans

${ }^{2}$ De l'avis de M CROZIER ET E FRIEDBERG, la stratégie est un ensemble cohérent de comportement qu'un acteur adopte en vue de préserver ses intérêts. La stratégie est orientée par les enjeux et zones d'incertitudes contrôlés par l'acteur social. De ce fait, toute stratégie est rationnelle aux yeux de l'acteur social qui l'utilise
} 


\section{Introduction}

L'évolution du passage de la colonisation à l'indépendance des pays d'Afrique subsaharien an accompagné de nombreux changements dans le système économique en général et en particulier dans le système de soin de santé de la population. En Côte d'Ivoire, par exemple, les périodes de 1960 (date de l'indépendance) à 1980, ont enregistrée une forte croissance économique avec un impact positif sur tous les secteurs parmi lesquels le domaine de la santé a significativement bénéficié. Un filet de structures sanitaires et de formation animé de personnel qualifié et de matériel adaptés s'est développé sur l'ensemble du territoire national. A cette époque, le financement de la santé de la population ivoirienne reposait essentiellement sur le seul budget de l'Etat (source). Les membres des différentes familles et/ou la communauté avaient recours au système de santé à n'importe qu'elle heure de la journée ou de la nuit, tout simplement parce que la prise en charge des soins était au compte de l'Etat. Cependant, les années 1980, marquées par la crise économique et les différentes programmes d'ajustement structurels ont été caractérisées par une réduction des recettes publiques. A partir de cette période, le budget de l'Etat ne pouvait plus supporter toutes les dépenses sanitaires de sa population. Dès lors, les populations sont convoquées à contribuer aux dépenses de leur propre santé. Cet désengagent de l'Etat dans l'accompagnement de la population dans la prise en charge des soins de santé expliquerait le développement d'autres pratiques des soins de santé.

Par ailleurs, certains comportements anti-productifs des agents de santé seront à l'origine d'autres formes de pratiques itinéraires thérapeutiques adoptées par les communautés. En effet, sur la base de la littérature relative au diagnostic des systèmes de santé, l'on constate que les usagers en Afrique sub-saharienne en générale subissent une panoplie de comportements inappropriés tels que l'insulte et brutalité, l'escroquerie, la négligence volontaire des soins, le mauvais accueil et la rétention d'information. Pour preuve, parlant des relations mères et centres de santé maternels, l'Unicef (1990) a relevé que : «parmi les causes qui dissuadent les femmes à recourir régulièrement aux centres de Protection Maternelle et Infantile (PMI), il y'a « le mauvais accueil, le ton employé, le langage impérieux sans respect de rapport ainé/cadets, le blocage linguistique, les contraintes administratives, l'environnement psychologique et l'indiscrétion du personnel de santé». Toutefois, bien que cette situation évoquée par la littérature conserve en partie leur validité pour de nombreuses familles africaines, ces informations ont en partie occulté les dynamiques thérapeutiques mises en œuvre à l'intérieur des familles.

Ainsi, la mise en œuvre officielle de la politique de contribution des populations aux dépenses de santé dans un contexte de paupérisation de la société, et associée aux comportements inappropriés des agents sont arrivés à produire progressivement des comportements thérapeutiques à l'intérieur des familles selon les conditions sociales et environnement culturel de chaque famille. Autrement dit, les familles ont elles-mêmes développé de nouvelles formes d'organisation de réponses thérapeutiques en vue d'assurer la santé de leurs membres. A preuve, les statistiques indiquent que le taux moyen de recours aux services de santé tourne autour de $20 \%$ malgré les efforts de l'Etat en vue d'améliorer l'accès aux soins publics de santé.. Le PNDS $^{3}$ confirme cette situation que le système de santé ivoirien qui, malgré ses infrastructures imposantes, est resté peu performant au regard des faibles taux de recours enregistrés depuis 1997 (PNDS, Tome 1). En outre, selon le

\footnotetext{
${ }^{3}$ Le plan National de Développement Sanitaire
} 
$\operatorname{MICS}^{4}$ (2006), le taux d'accès aux services publics de santé de la Côte d'Ivoire est l'un des taux les plus bas de la sous-région.

A partir de ces constats la présente étude a pour objectif d'appréhender la dynamique sociale et les perceptions qui structurent les pratiques thérapeutiques à l'intérieur des familles du milieu étudié et les résultats de santé qui en découle. Autrement dit, l'étude vise à apporter des éléments de compréhension sur les formes et mécanismes d'organisation thérapeutique des familles de la communauté Abbey et les résultats de santé obtenus. Nous avions procédé à l'observation des familles concernées par l'étude, les modes d'interprétations des normes sociales en matière de soins thérapeutiques. Cette réflexion favorise l'interrogation suivante: Comment les populations construisent le sens et la signification de leurs pratiques, dans un contexte soumis à la pauvreté et à l'émergence des pratiques de soins juxtaposés?

\section{Matériels Et Method}

\section{Matériel}

L'article étudie l'évolution des pratiques thérapeutiques familiales depuis le passage de la politique de gratuité des soins à la décision de contribution des ménages aux coûts de recours aux soins de santé en côte d'Ivoire. Ainsi, méthodologiquement, l'article s'appuie sur une approche qualitative à visé compréhensive axée sur une compréhension fine du contexte familial de dynamique thérapeutique.

La première phase a consisté à l'exploration documentaire et l'observation participante du milieu étudié. Cette phase a permis de décrire de façon structurante la réalité des faits thérapeutiques et de leur dynamisme à l'intérieur des familles cibles et dans l'environnement social du peuple Abbey. La période d'observation de huit (08) mois a été structurée par une grille d'observation formalisée dans un cahier. Dans ce cahier appelé journal de terrain, nous avons noté ce que nous avons vu, entendu, ressenti sur le fonctionnement thérapeutique des familles cibles durant tout le long de ce processus. Les éléments observés s'articulaient autour, des formes et mécanismes d'organisation thérapeutique des familles et les résultats sociaux de santé qui en découlent. Ainsi, nous avons pu cerner les modes d'interprétations et de réinterprétations des normes sociales en matière de soins thérapeutique.

La deuxième phase a été les entretiens semi-structurés auprès des acteurs cibles. L'approche développée ici s'inscrit dans un intérêt plus global pour les configurations familiales en relation dans le système de soin, entre des acteurs familiaux ayant des rapports diversifiés à la santé et/ou la maladie (diagnostic, recherche médicale, etc). Le guide d'entretien a été construit autour de l'interrogation suivante : Comment ils construisent le sens et la signification de leurs pratiques en matière des soins de santé dans un contexte soumis à la pauvreté et à l'émergence des pratiques de soins juxtaposés ? La dynamique des pratiques thérapeutiques des familles peuvent être plus ou moins éloignée des principes éthiques ou entrée en contradiction avec les recommandations des autorités sanitaires. Pour comprendre cette dynamique en ballotage entre modernité et tradition, il est nécessaire d'interroger les acteurs clés ${ }^{5}$ des familles sur leur expérience, leurs logiques et les références (endogènes or exogènes) qu'ils mobilisent.

\footnotetext{
${ }^{4}$ Enquête à indicateurs multiples

${ }^{5}$ Les personnes responsables ou les décideurs des itinéraires thérapeutiques des membres du ménage
} 


\section{Participants}

Les entretiens ont été réalisés auprès de 22 personnes issues de 7 familles différentes. Pour des raisons de la configuration sociale non-maitrisé des familles le nombre d'individus à interroger n'a pas été fixé. Il s'est agi d'interroger dans chaque famille le chef de famille, la mère, la grande mère et/ou grand-père et le fils ainé ou d'une tierce personne de la famille dont l'âge est compris entre 18 et 45 ans. Compte tenu de la taille des familles et de l'intérêt du sujet, ces entretiens individuels se substituaient en des réunions dirigées de groupe avec les personnes clés visées. Le tableau cidessous donne la composition exacte des personnes interrogées par famille.

Tableau 1: Composition des personnes interrogées par famille

\begin{tabular}{|c|c|c|c|c|}
\hline Famille & Composition/famille & Profil des membres enquêtés & Nbre/Pers/enqtée & Total \\
\hline \multirow[t]{4}{*}{$\mathrm{F}_{1}$} & \multirow[t]{4}{*}{7} & $\mathrm{CF}$ & 1 & \multirow{4}{*}{4} \\
\hline & & $\mathrm{M}$ & 1 & \\
\hline & & Fils ainé & 1 & \\
\hline & & GM & 1 & \\
\hline \multirow[t]{4}{*}{$\mathrm{F}_{2}$} & \multirow[t]{4}{*}{9} & $\mathrm{CF}$ & 1 & \multirow{4}{*}{4} \\
\hline & & $\mathrm{M}$ & 1 & \\
\hline & & GM & 1 & \\
\hline & & GP & 1 & \\
\hline \multirow[t]{3}{*}{$\mathrm{F}_{3}$} & \multirow[t]{3}{*}{5} & $\mathrm{CF}$ & 1 & \multirow{3}{*}{2} \\
\hline & & M & 1 & \\
\hline & & Frère du CF & 1 & \\
\hline \multirow[t]{3}{*}{$\mathrm{F}_{4}$} & \multirow[t]{3}{*}{4} & $\mathrm{CF}$ & 1 & \multirow{3}{*}{3} \\
\hline & & $\mathrm{M}$ & 1 & \\
\hline & & Belle mère du CF & 1 & \\
\hline \multirow[t]{4}{*}{$\mathrm{F}_{5}$} & \multirow[t]{4}{*}{8} & $\mathrm{CF}$ & 1 & \multirow{4}{*}{4} \\
\hline & & $\mathrm{M}$ & 1 & \\
\hline & & GM & 1 & \\
\hline & & GP & 1 & \\
\hline \multirow[t]{3}{*}{$\mathrm{F}_{6}$} & \multirow[t]{3}{*}{5} & $\mathrm{CF}$ & 1 & \multirow{3}{*}{3} \\
\hline & & $\mathrm{M}$ & 1 & \\
\hline & & GP & 1 & \\
\hline \multirow[t]{2}{*}{$\overline{\mathrm{F}_{7}}$} & \multirow[t]{2}{*}{4} & $\mathrm{CF}$ & 1 & \multirow{2}{*}{2} \\
\hline & & $\mathrm{M}$ & 1 & \\
\hline Total en & quêté & & & 22 \\
\hline
\end{tabular}

Légende: $\mathrm{GM}=$ grand-mère, $\mathrm{GP}=$ grand père, $\mathrm{CF}=$ chef de famille; $\mathrm{M}=$ mère

Ces différents acteurs cibles ont des connaissances ou un lien profond avec des pratiques thérapeutiques à l'intérieur de leur famille respective. Les familles ont été choisies par réseau ou boule de neige et sur la base de l'expérience thérapeutique et du mode de structuration sociale de chacune d'entre elle. De ce point de vu, trois catégories de familles (économiquement faible, économiquement moyenne et économiquement bonne ou acceptable) ont été choisie dans leur façon de construire la santé de leur membre. 
Des entretiens aussi ont été réalisés auprès de cinq (05) vendeuses de médicaments de la rue et de cinq (05) vendeuses des plantes médicinales. Ces échanges ont permis de savoir le profil des personnes qui fréquentent ces marchés de vente de médicaments de la rue supposés «moins chers» et des plantes médicinales. Auprès de ces vendeuses, nous avons compris l'idée selon laquelle les populations s'intéressent à ce marché ou se soignent à partir de ce marché de médicament non conventionnels.

Les données recueillies ont fait l'objet d'analyse de contenu des guides d'entretien. A partir de cette grille d'analyse, les éléments de réponse sur les normes familiales en matière de construction de la santé ou l'orientation pours les soins ont été suffisamment cernés. Cette approche a permis de comprendre à travers les propos, les schémas et les comportements des acteurs interrogés, la dynamique thérapeutique à l'intérieur des familles.

\subsection{Dynamique Thérapeutique Familiale Dominée Par Les Pratiques Curatives De Soin}

Depuis la fin de la période de gratuité initiée au lendemain de l'indépendance du pays, la dynamique curative des soins s'intensifie à l'intérieur des familles. Autrement dit, la dynamique thérapeutique à l'intérieur des familles se caractérise de plus en plus par une multiplicité de pratiques curatives de soins avec une juxtaposition des soins mobilisables à cet effet. Cette juxtaposition thérapeutique reste néanmoins en ballotage entre modernisme et traditionalisme. Ainsi, sur la base des entretiens et observations, il a été constaté que dans la quasi-totalité des familles enquêtées, la structuration de la santé et/ou la déconstruction de la maladie est avant tout une scène d'échange thérapeutique ou de soutien thérapeutique des membres de la familles, voisins et amis constitue l'élément social qui renforce la confiance et le moral du malade. En effet, l'un des éléments qui renforce l'idée selon laquelle les pratiques thérapeutiques curatives emportent sur celles dites préventives à l'intérieur des familles est la qualité et la place qu'occupe dans la conscience du malade et de sa famille, le sens des visites symboliques des voisins, de l'entourage et des amis. De l'avis de 63,23\% des enquêtés, les visites accompagnées de prières ou de vœux des voisins, amis ou entourage constitue le premier élément de guérison du malade pour au moins deux grandes raisons.

Premièrement, les visites de l'entourage, voisins, et amis etc constituent pour la majorité des enquêtés, un symbole qui décrit la qualité des relations sociales et culturelles entre la famille du malade et/ou du malade et son entourage. De ce point de vu, la maladie constitue un indicateur permettant de tester le niveau de relation avec sa communauté. Considéré comme une référence aux valeurs morales et socio-culturelles qui sous-tendent toutes sociétés dites primitives, ce symbolisme thérapeutique constitue la source culturelle de légitimation des pratiques curatives dans les familles. Autrement dit, le modèle curatif de soin dans lequel s'inscrit la majorité des familles s'explique dans un premier temps de façon inconsciente par le souci de tester son niveau de relation communautaire de la famille et/ou du malade.

En réalité, dans nos sociétés traditionnelles, ou le collectif emporte sur l'individualisme, ne pas recevoir de visite des siens ou des proches lors de sa maladie est un symbole de mauvaise relation communautaire du malade ou de la famille du malade. C'est pourquoi, il n'est pas rare d'entendre des familles dirent : "entretenir la relation communautaire est mieux que l'argent et tout ». 
Or de telles idées ou visions, même si elles trouvent leur validité dans certains domaines de la vie, sont destinées à anesthésier la conscience sanitaire ou thérapeutique du malade et de sa famille. Ainsi, comme résultat, le malade décède le plus souvent de maladie facilement curable faute de méthode préventive.

Deuxièmement, dans l'imaginaire populaire des populations enquêtées, les vœux ou prière que les visiteurs font à la famille pour la reconstruction rapide du malade est source de guérison. Par exemple, de l'avis d'un enquêté, les prières du genre «que Dieu Guérisse le malade », "que Dieu renforce la santé du malade et de sa famille » sont vite exhaussées par le seigneur, détenteur de la santé, la maladie et de la guérison. Les prières des uns et des autres dans lesquels s'affirment les rapports communautaires acquièrent donc la forme d'un rapport social thérapeutique a visé curative. L'approbation de ces normes et pratiques dans les consciences collectives et individuelles des enquêtés n'est pas donnée une fois pour toute, mais elles sont le produit d'une construction traditionnelle. En leurs accordant à un moment donné des valeurs particulières, la reconnaissance communautaire contribue à les faire exister de manière permanente sur la scène médicale.

En réalité, la logique à l'œuvre sur la scène médicale des familles est une logique de « saiyè noufou» ou «kain-nain-ya» respectivement en langue Abbey et malinké qui signifie de «curative » ou encore de «guérison ». Les pratiques thérapeutiques à l'intérieur des familles, qu'elles soient communautairement formalisées ou non sont éloignées de tout exercice médical préventive. Alors que la prévention constitue un moyen permettant de renforcer le socle de protection de l'individu. Ne dit-on pas que 'prévenir vaut mieux que guérir'! Ainsi, l'analyse de contenu des discours des enquêtés laisse entrevoir que les pratiques curatives de soin conditionnent bien les rapports médicaux à l'intérieur et à l'extérieur de la sphère familiale, et ce système de pratique résiste jusque-là aux campagnes de politiques préventives prônées par les autorités sanitaires. On peut en juger par la proportion importante (plus de 50\%) des propos affichés des enquêtés des différentes catégories sociales familiales à ce sujet.

A l'analyse, l'éloignement des individus des pratiques thérapeutiques préventives prônées par les autorités sanitaires fait partie de stratégie d'acteur dont parle Michel CROZIER ${ }^{6}$ (1977), soit pour contourner les dépenses financières, soit pour donner sens à un évènement jugé pas trop défavorable pour eux ou soit pour atteindre un ordre socio-culturel non communicable.

Ces faits tantôt observés, permettent d'objectiver l'hypothèse suivante ; 'si à l'intérieur des familles, les pratiques curatives emportent sur celles dites préventives, c'est parce que le passé ou l'histoire médicale des familles, fondée sur la tradition, est restée jusque-là la boussole sociale en matière de construction de la santé' '. Un enquêté confirme cette idée lorsqu'il dit:

«Nos grands parents ne prévenaient pas de maladie, ils les traient de façon curative, mais ils vivaient plus longtemps que ceux qui parlent de méthode préventive de la maladie aujourd'hui. $C$ 'est quand la maladie s'installe bien dans le corps qu'on arrive à la diagnostiquée bien et à trouver le bon remède ».

\footnotetext{
${ }^{6}$ De l'avis de M CROZIER ET E FRIEDBERG, la stratégie est un ensemble cohérent de comportement qu'un acteur adopte en vue de préserver ses intérêts. La stratégie est orientée par les enjeux et zones d'incertitudes contrôlés par l'acteur social. De ce fait, toute stratégie est rationnelle aux yeux de l'acteur social qui l'utilise
} 
L'analyse du discours de l'enquêté laisse entrevoir que dans l'imaginaire collective et/ou individuelle des enquêtés, les maladies doivent être traitées à condition qu'elles se manifestent concrètement sur une longue période. Car c'est sur une longue période (voir une semaine ou deux semaines selon leur avis) que la famille du malade ou l'agent de santé,- quand l'état du malade devient sérieux- arrive à bien diagnostiquer la maladie et à trouver le remède idoine. C'est pourquoi la littérature (Helman, 2004 ; Sylvie ., 2004) soutient que dans les sociétés dites traditionnelles le traitement de la maladie donne lieu à des savoirs et des significations plus ou moins formalisés, stabilisés, porteurs de représentations, de normes et de valeurs. En règle générale, il y'a un grand fossé entre la méthode curative et la méthode préventive dans la dynamique thérapeutique des familles, ce qui n'est pas sans conséquence sur la santé des membres. En cas d'épisode morbide chez un agent social, les acteurs mettent en œuvre des stratégies conformes à leur 'rationalité' au double sens Wébérien de rapport aux fins et aux valeurs. Le recours le plus immédiat et le plus sollicité est, selon nos investigations le «saivirigbé » ou la médecine traditionnelle ou guérisseurs (dans l'appellation linguistique du peuple Abbey). Une approche de soins ou de médication structurée de plante (feuilles, racines, tiges, fruits...), décoction, produit à base de kaolin, poire à lavement, etc. Il le fait soit en faisant appel à ses propres connaissances endogènes ou exogènes, soit avec l'aide du groupe organisateur de la thérapie, en général des personnes âgées qui ont une certaine expérience de la pharmacopée propre à la tradition.

Si cette première stratégie thérapeutique mobilisée ne produit pas la guérison chez l'individu malade, ce dernier est conduit au centre de santé chinois ${ }^{7}$, pour son traitement. Mais, lorsque les symptômes de la maladie persistent, l'éventail des comportements s'ouvre de telle façon que la maladie devient un phénomène social dont l'explication, l'interprétation et la thérapie mettent en jeu les représentations cosmologiques et l'organisation socioculturelle. C'est-à-dire que la famille revient à la pratique médicale traditionnelle et ainsi de suite jusqu'à la guérison ou le décès du malade.

Memel Fôté confirme cette affirmation lorsqu'il dit que l'anthropologie de la santé témoigne de deux faits de portée presque universelle: le premier, c'est que dans les sociétés connues, les malades en quête de thérapie, au sens de leur vision du monde suivent une diversité d'itinéraires thérapeutiques à leur disposition; le second fait est que ces itinéraires appartiennent soit à un même système médical, soit à deux ou plusieurs systèmes d'origines différentes (Memel Fôté, 1998).

En conclusion, l'on retient l'existence dans les familles, d'une organisation de la maladie et de la thérapie par un ensemble de parents proches. La forme d'organisation axée sur un système curatif de soins constitue un aspect central de la dynamique familiale en matière de santé. En effet, chaque fois qu'un agent social est malade, un groupe se constitue autour de lui. Différents parents (maternels et paternels) et éventuellement leurs associés s'unissent dans le but d'examiner minutieusement les informations, d'apporter leur soutien moral, de prendre les décisions qui s'imposent et de mettre au point les détails de la consultation thérapeutique.

\subsection{Diversité Et Instabilité Du Contenu Du Panier Pharmaceutique Familial}

\footnotetext{
${ }^{7}$ Depuis ces deux décennies, la médecine chinoise est bien implantée sur tout le territoire ivoirien
} 
En prélude à ce chapitre, il est bien de préciser sur la base des observations qu'il n'existe pas de société au degré zéro de la connaissance médicale. Dans toutes les sociétés humaines les acteurs ont élaboré des cadres logiques non pas pour atteindre une connaissance vérifiable et communicable, mais pour mettre en ordre leur santé. D'ailleurs, depuis le commencement de la vie sur terre, la maladie existe en tant que phénomène biologique et social. Ce qui veut dire que l'être social n'a jamais été inactive vis-à-vis de la malade. Elle a toujours été en quête de cause, de sens, de thérapie ou de remède pour ainsi arriver à contrecarrer ces maux.

Ce chapitre s'inscrit dans cette démarche et vise à questionner le contenu de la boîte familiale à pharmacie tout en se préoccupant des logiques de soin, de la cohérence de système de diagnostics et de traitement de la maladie des acteurs à la lumière du contexte tantôt traditionnel, tantôt moderne dans lequel ils s'insèrent. Ainsi, le questionnement du panier de soins réalisés laisse entrevoir que les groupes familiaux interviewés, loin de se limiter à la sphère médicale recommandée par les autorités sanitaire sont significativement influencés par des pratiques thérapeutiques relevant de la tradition. C'est-à-dire que le panier pharmaceutique est largement structuré des produits thérapeutiques logés dans le moule de la tradition et des produits thérapeutiques relevant du 'sens commun' appelés les médicaments de la rue.

Toutefois, l'un des points les plus importants réside dans tout ce qui a été recueilli comme informations, l'existence de panier pharmaceutique "symbolique ou non'" dans toutes les familles visitées. En effet, les familles enquêtées, notamment celles classées parmi les familles économiquement faible et moyen attestent avoir un panier pharmaceutique familiale au sein de leur lieu d'habitation ou de leur espace social. A l'analyse, la boîte pharmaceutique des familles est dans la main des "saivirigbe'" et des vendeuses que les populations appellent "les docteurs de rue" ou encore les vendeuses des médicaments du "sens commun" avec qui elles partagent le plus souvent les rapports de voisinage. Ce sont donc, ces vendeuses de médicaments de la rue qui approvisionnent dans les quartiers, les marchés, les grains ${ }^{8}$ et les domiciles familiales de façon quotidienne (matin et soir) en plus des soins venant des médecins traditionnels « saivirigbé ».

Ainsi, sur la base des enquêtes réalisées, on constate une similitude de pratiques d'itinéraires pour les soins au sein de famille qu'elle soit économiquement faible ou moyen, et même acceptable ou bonne.

Pour le groupe de famille économiquement faible et moyen (79\%), le premier groupe de produit de la boîte à pharmacie est composée de médicament du 'sens commun' ou encore de médicaments de la rue''. Et dans l'imaginaire collective des familles, les vendeuses de ces produits illégaux sont les substituts des médecins formés à grand frais par l'Etat de Côte d'Ivoire. L'automédication représente une partie intégrante dans le processus de structuration du panier de soins familiaux. Ainsi, les produits les plus sollicités par les populations qui fréquentent ces marchés sont présentés dans le tableau ci-dessous.

Tableau 2: Liste du contenu du panier familial à pharmacie

\footnotetext{
${ }^{8}$ Un lieu ou espace de rencontre, de causerie et d'échange de regroupement de personnes qui se partagent des débats sur des idées touchant toutes les dimensions de la société. Le grain a vu le jour pendant les périodes des crises depuis 2002
} 


\begin{tabular}{|c|c|c|c|}
\hline $\begin{array}{l}\text { Nom commercial du } \\
\text { produit }\end{array}$ & $\begin{array}{l}\text { Dosage } \\
(\mathrm{ml})\end{array}$ & $\begin{array}{l}\text { Prix/unité } \\
\text { (FCFA) }\end{array}$ & $\begin{array}{l}\begin{array}{l}\text { Indications } \\
\text { commun }\end{array} \\
\end{array}$ \\
\hline \multirow[t]{2}{*}{ Paracetamol } & \multirow[t]{2}{*}{500} & \multirow[t]{2}{*}{50} & Contre les maux de tête \\
\hline & & & Douleur dans le corps \\
\hline Daga & 500 & 50 & $\begin{array}{l}\text { Combat toutes sortes de maladie dans } \\
\text { le corps }\end{array}$ \\
\hline Epice & pas inscrit & 50 & Lutte fortement contre la céphalée \\
\hline Wourou fatô & pas inscrit & 50 & $\begin{array}{l}\text { Combat toutes sortes de maladie dans } \\
\text { le corps }\end{array}$ \\
\hline Sassaborô & 300 & 25 & Migraine et maux de ventre \\
\hline Dabôssi & 5 & 20 & plaie de bouche et de ventre \\
\hline Beflavine & 10 & 75 & Plaie de ventre \\
\hline Chauffeurdjè & pas inscrit & 50 & $\begin{array}{l}\text { Puissance sexuelle, fièvre, grippe } \\
\text { rhume }\end{array}$ \\
\hline Royal & 200 & 50 & Ulcère \\
\hline Bisacodyl & 100 & 300 & Paludisme \\
\hline Tanjol & 100 & 300 & Paludisme \\
\hline $\begin{array}{l}\text { Mousokôrôni-ka } \\
\text { ballontant }\end{array}$ & 250 & 50 & $\begin{array}{l}\text { Faiblesse sexuelle, fatigue générale, } \\
\text { paludisme }\end{array}$ \\
\hline
\end{tabular}

Source: données d'enquête du terrain, 2017

Cette boîte à pharmacie est composée des produits non recommandés par les autorités sanitaires. Et pourtant, ces produits constituent l'objet de recours pour la majorité des familles. A l'analyse, c'est le coût d'accès et la facilité de renouveler sa boîte à pharmacie qui orientent plus ces familles acculées par la pauvreté vers ces produits. Memon et Kassoum. (2016) affirment dans leur étude intitulé « représentation sociale et recours au système de santé publique au sud de la Côte d'Ivoire : une analyse à partir des données empiriques » que le recours à ces types de produits très accessibles mais dangereux pour la santé est lié à «la monétarisation des rapports médicaux. En effet, dans l'imaginaire des personnes [...] l'hôpital est présenté non pas d'abord comme un lieu où l'on reçoit des soins dans des conditions d'accueil acceptable mais comme un marché 'Gouro d'Adjamé ', où l'on discute du prix de biens marchands. Sur cet espace social situé au cœur de la commune d'Adjamé, district d'Abidjan, 'l'argent' est au centre des rapports sociaux qui se construisent et se déconstruisent. Cette caractéristique de 'marché gouro' que les usagers attribuent aux services de santé publique est arrivée à « fétichiser » (rendre mystérieux) la réalité des soins hospitaliers même en période de gratuité ciblée. Dans ces conditions, l'hôpital ne sera attentif aux réalités anthropologiques que sont le malade et sa maladie que si et seulement si l'usager s'inscrit dans ce nouveau type de rapport économique ». La vente et l'administration de ces produits, constituant du panier pharmaceutique des familles sont tenues par des femmes qui n'ont pas acquis de formation.

Le deuxième groupe de produit du panier pharmaceutique est structuré autour du 'saivirigbé". "Saivirigbé" signifie dans la linguistique Abbey (langue autochtone de la zone d'étude) la médecine de nos ancêtres appelée communément médecine traditionnelle. Il s'agit ici du système

9 Le marché Gouro est le plus grand marché de la commune d'Adjamé du District d'Abidjan. C'est un espace d'échange de bien et d'objet de valeur. 
thérapeutique reposant sur des savoirs dits traditionnels délivrés par des thérapeutiques tradipraticiens à travers le transfert de connaissances endogènes et exogènes, des groupes de guérisseurs dont seuls les membres ont des dons et des pouvoirs de guérison à partir du monde spirituel, des féticheurs, des guides religieux appelés « marabouts » à travers le 'Nanssidji', etc...

Ce qui paraît intéressant de voir est que les familles de l'espace d'étude comme le montre Dacher (1992) pour le peuple Gouin, Faizang (1986) pour le peuple Bissa, et Jacob (1988) pour le peuple Winyz, expriment les notions de bonne santé et de mauvaise santé en terme de "frais-froidhumide/sec-chaud». Ainsi, on dira de quelqu'un qu'il est malade en ce terme en Abbey "sâyènoufô » (son corps est chaud) et "Orogba'" (maladie) qui signifie dans le contexte socioculturel des familles la situation de celui qui se trouve momentanément affecté de corps ou d'esprit. En effet, il faut non seulement chercher à le guérir de l'affection dont il est frappée en déterminant au préalable, la nature du mal en cause, mais encore, au cours des soins qui peuvent parfois être longs. Ces expressions « Orogba », « sâyènoufô », « sâyaimou » sont traitées par les « khèlèwossô » à base de plante. Sur la base des observations et entretiens, il convient de retenir que ces plantes constituent le remède d'une panoplie de maladie (voir Tableau 3). A cet effet, précisons que dans la localité étudiée, le nombre de thérapeutes traditionnels ou « kpèlèwo » en activité est important comme nous l'avons signifié un peu plus haut.

Tableau 3: Mode de traitement de quelque maladie selon la thérapie traditionnelle

\begin{tabular}{|c|c|c|c|}
\hline Plantes & phénomènes morbides & $\begin{array}{l}\text { Partie } \\
\text { utilisée }\end{array}$ & $\begin{array}{l}\text { Modes } \\
\text { d'administration }\end{array}$ \\
\hline sébélilli & $\begin{array}{l}\text { Asthme } \\
\text { Choléra } \\
\text { Hémorragie après accouchement }\end{array}$ & $\begin{array}{l}\mathrm{R} \\
\mathrm{R} \\
\mathrm{R} \\
\end{array}$ & $\begin{array}{l}\text { Boisson } \\
\text { Purge } \\
\text { Boisson }\end{array}$ \\
\hline $\begin{array}{l}\text { kolokolo- } \\
\text { bourou }\end{array}$ & $\begin{array}{l}\text { Rhumatisme } \\
\text { Stimulant }\end{array}$ & $\begin{array}{l}\mathrm{F} \\
\mathrm{F}\end{array}$ & $\begin{array}{l}\text { Friction } \\
\text { Purge }\end{array}$ \\
\hline kobétourou & $\begin{array}{l}\text { Diabète } \\
\text { Diarrhée } \\
\text { Paludisme }\end{array}$ & $\begin{array}{l}\mathrm{F} \\
\mathrm{F} \\
\mathrm{F}\end{array}$ & $\begin{array}{l}\text { Boisson } \\
\text { Purge } \\
\text { Boisson, lavage }\end{array}$ \\
\hline $\begin{array}{l}\text { coffea } \\
\text { canephora }\end{array}$ & Hypertension & $\mathrm{F}$ & Boisson, lavage \\
\hline dabadaba & Paludisme & $\mathrm{F}$ & Boisson, lavage \\
\hline zababourou & $\begin{array}{l}\text { Fièvre } \\
\text { Stérilité féminine }\end{array}$ & $\begin{array}{l}\mathrm{F} \\
\mathrm{F} \\
\end{array}$ & $\begin{array}{l}\text { Boisson } \\
\text { Boisson } \\
\end{array}$ \\
\hline zababourou & $\begin{array}{l}\text { Fièvre } \\
\text { hypertension } \\
\text { Contraception } \\
\end{array}$ & $\begin{array}{l}\mathrm{F} \\
\mathrm{F} \\
\mathrm{F}\end{array}$ & $\begin{array}{l}\text { Boisson, lavage } \\
\text { Boisson } \\
\text { Boisson } \\
\end{array}$ \\
\hline kobétourou & $\begin{array}{lll}\begin{array}{l}\text { Rhumatisme, } \\
\text { sorcellerie }\end{array} & \text { Contre la } \\
\end{array}$ & $\mathrm{H}$ & Pommadé \\
\hline
\end{tabular}

Légende: $\mathrm{F}=$ Feuille; $\mathrm{R}=$ racine, $\mathrm{H}=$ huile

Cette gamme de produits traditionnels ou des plantes sont généralement vendues sur le marché auprès des vendeuses qui approvisionnent les particuliers et des guérisseurs ou « khèlèwossô » qui en font la demande mais aussi sont recherchées en brousse par les demandeurs eux-mêmes pour des raisons de conservation. Des particuliers s'adressent directement à elles, sans passer au 
préalable par un guérisseur. Elles occupent très souvent la brousse du département à la recherche des plantes. Elles affirment que l'apparition ou la résurgence de maladies est recrudescente, de ce fait qu'elles vendaient davantage aussi bien en volume qu'en diversités espèces commercialisées. De l'analyse, on retient que ce panier symbolique de pharmacie des familles maintenues dans un statut socioéconomique faible et moyen est caractérisé entièrement par les deux types de produits qui sont « les médicaments de la rue et saivirigbé »

Pour les familles économiquement bonne ou acceptable, (21\%), une bonne partie de la boîte à pharmacie est structurée de médicaments de pharmacie moderne et conventionnelle. Ce sont les produits comme le Thermomètre, Ca-C1000, les comprimés Efferalgan, des vitamines tel que Ecsodyne, des produits de massages, etc. Il existe aussi des médicaments de la rue qu'ils achètent auprès des vendeuses de proximité dans leur environnement social direct, soit à la place appelée «Roxi » situé au grand marché d'Adjamé (une commune de commerce de la ville d'Abidjan à environ quatre-vingt kilomètres d'Agboville le site d'étude). De leur avis, dans ce marché, on y trouve l'équivalent de plusieurs produits pharmaceutiques à des tarifs très accessibles. De même, ils estiment que les médicaments de qualité des pharmacies reconnues par les autorités se retrouvent sur ces marchés à bon marché. Alors que dans les pharmacies modernes et conventionnelles, les mêmes médicaments ne sont pas accessibles à cause des prix élevés ou par manque de stock. Aux dires de ces personnes, les médicaments de la rue comblent souvent le vide.

Par ailleurs, il faut noter que malgré l'utilisation des produits de la pharmacie conventionnelle, les membres de cette catégorie de famille font aussi de la juxtaposition de pratiques thérapeutiques. En effet, ils fréquentent aussi les «saivirigbé », des groupes de tradipraticiens pour des soins à base des plantes et spirituels quand les maladies dépasse leur compréhension. A la recherche d'une réponse ou d'une solution pour les soins ils n'hésitent pas s'orienter vers la médecine traditionnelle afin de renforcer celles administrées par la médecine modernes.

En somme, l'on retient que pour cette catégorie de famille, la pratique de l'automédication se partage avec les médicaments modernes dont une part non négligeable provient de source non recommandé.

\section{Conclusion}

Toutes les familles, de façon consciente ou inconsciente, développent des politiques en matière de santé de ses membres. A ce niveau, la démarche adoptée a été de poser les bases méthodologiques et théoriques de l'étude. Dans les débats sociologiques opposant les auteurs de la démarche compréhensive (Weber, 1997) à ceux de la démarche explicative (Durkeime, 1950), Bourdieu (1993) souligne que « comprendre et expliquer ne font qu'un ». En application de ce fondement, l'approche de l'étude qui a privilégié les entretiens et observations ont cherché à expliquer et comprendre, d'une part, la dynamique thérapeutique à l'œuvre à l'intérieur des familles et de l'autre part, l'évolution des pratiques thérapeutiques au sein des familles depuis le passage de la gratuité des soins à la politique de contribution des ménages pour leur soins de santé en côte d'Ivoire.

Les faits observés et le fonctionnement social des différents acteurs clés interrogés ont permis de dégager des traits structurants et pertinents dans la dynamique de soin à l'intérieur des familles. Ainsi, la perspective compréhensive menée dans la présente étude a permis de savoir que la 
dynamique thérapeutique à l'intérieur des familles est dominée par les pratiques curatives de soins avec une juxtaposition des soins mobilisables (médecine moderne et traditionnelle, l'automédication). Cette domination du curatif sur le préventif est destinée à anesthésier la conscience sanitaire ou thérapeutique du malade et de sa famille. En outre, l'étude a révélé que le panier pharmaceutique est largement structuré de produits thérapeutiques logés dans le modèle de la tradition et des produits thérapeutiques relevant du 'sens commun' (médicament de la rue). La conséquence qui découle de toutes ces pratiques de santé à l'intérieur des familles est l'observation du décès de l'individu par une maladie facilement curable.

\section{Remerciements}

Nous voudrions remercier tous ceux qui ont contribué à travers leurs idées et commentaires à consolider le présent article, notamment Dr N'dri Kouamé Abou, Dr Sangaré Moussa. avec qui nous travaillons continuellement. Nos remerciements vont à l'endroit des leaders communautaires et les differents chefs de familles pour leur collaboration pendant la phase de collecte des données sur le terrain. A Mlle Bamba Ami et Dosso Nathali, étudiantes en Masters de sociologie de l'Université Peleforo Gon Coulibaly-Korhogo, pour leur contribution à la collecte des données. Nous remercions particulièrement la Direction du Centre Ivoirien de Recherches Economiques et Sociales (CIRES) pour la collaboration de travail qu'elle nous offre.

\section{Conseils Généraux Sur L'écriture Des Résultats De La Recherche}

L'évolution du passage de l'indépendance à l'avènement du multipartisme de la majorité des pays d'Afrique subsaharien en général et plus particulièrement en Côte d'Ivoire a accompagné de nombreux changements dans le système de soin de santé à l'intérieur des familles. A travers le présent article, nous avons cherché à comprendre les formes et expressions des dynamiques thérapeutiques à l'œuvre à l'intérieur des familles. Comme les familles ont plus de penchant aux pratiques médicales traditionnelles, l'étude recommande une collaboration objective entre les deux formes de médecines (médecine moderne et traditionnelle). Nous pensons que cette collaboration permettra de réduire significativement les dangers liés aux pratiques médicales traditionnelles des familles.

\section{Références (bibliographiques)}

[1] Antoine Angaté. (2002). La revalorisation de la médecine traditionnelle, édition CEDA, pp17-49.

[2] Fainzang Sylvie. (2001). L'anthropologie médicale dans les sociétés occidentales. Récents développements et nouvelles problématiques. Sci Soc Santé 1-28P

[3] Harris Memel Foté. (1998). La santé, la maladie et les médecines en Afrique, une approche anthropologique, collection sociétés africaines et diaspora ; le Harmattan, 210p

[4] Jacqueline Andoche. (1988). L'interprétation populaire de la maladie et de la guérison a l'Ile de la réunion, Sciences Sociales et Santé, Vol VI, n ${ }^{\circ} 3-4$, Novembre 1988, Paris

[5] Fainzang Sylvie. (2004). Entre pratique symbolique et recours thérapeutique; les problématiques d'un itinéraire de recherche, centre de recherche médecine, science, santé et société.

[6] Lévi-Strauss Claude., (1958). «L'efficacité symbolique », in Anthropologie structurale I. Paris: Plon, pp. 205-226. 
[7] Louis Paul Aujoulat (1969). Santé et développement en Afrique, institut International d'Administration publique, Armand Colin, Paris 1969

[8] Memon. Fofana \& Kassoum. Traoré. (2016). Représentation sociale et recours au système de santé publique au sud de la Côte d'Ivoire : Une analyse à partir des données empiriques; journal of socials sciences Research ; Council for innovative Research; http://cirworld.com/journals/index.php/jssr/issue/view/542 ISSN 2321-1091 volume 10 Number 2; May 2016, P.P 2039-2046

[9] Cantrelle Pierre, Locoh Thérèse. (1990), Facteurs culturels et sociaux de la santé en Afrique de l'ouest. Les dossiers du Ceped.

[10] Sow Khoudia., (2013). De l'exception à la «normalisation»: Anthropologie de la santé reproductive confrontée au VIH au Sénégal, Thèse de Doctorat en Anthropologie, Aix-enProvence, Aix-Marseille Université, 262 p.

[11] Badji Mouhamed Ahmed, Ndione Albert Gautier, Desclaux Alice, Sow Khoudia., (2015). « Vécu de la "surveillance communautaire" par les soignants contacts au Sénégal », Conférence Ebodakar: Épidémie d'Ebola en Afrique de l'ouest. Approches ethno-sociales comparées, Dakar, 19-21 mai 2015.

[12] Thérèse Locoh (1995). Famille africaine, population et qualité de la vie, Centre français sur la population et le développement (Ceped), dossier $n^{\circ} 31$

\footnotetext{
*Corresponding author.

E-mail address: traorekassfr@ yahoo.fr/fofanamemon20@yahoo.fr
} 